University of St. Thomas, Minnesota

UST Research Online

$1-1-1995$

Principal Submatrices, Geometric Multiplicities, and Structured Eigenvectors

Charles R. Johnson

Brenda K. Kroschel

University of St. Thomas, Minnesota, bkkroschel@stthomas.edu

Follow this and additional works at: https://ir.stthomas.edu/cas_mathpub

Part of the Mathematics Commons

This Article is brought to you for free and open access by the Mathematics at UST Research Online. It has been accepted for inclusion in Mathematics Faculty Publications \& Data by an authorized administrator of UST Research Online. For more information, please contact asle4660@stthomas.edu. 


\title{
PRINCIPAL SUBMATRICES, GEOMETRIC MULTIPLICITIES, AND STRUCTURED EIGENVECTORS *
}

\author{
CHARLES R. JOHNSON ${ }^{\dagger}$ AND BRENDA K. KROSCHEL ${ }^{\dagger}$
}

\begin{abstract}
It is a straightforward matrix calculation that if $\lambda$ is an eigenvalue of $A, x$ an associated eigenvector and $\alpha$ the set of positions in which $x$ has nonzero entries, then $\lambda$ is also an eigenvalue of the submatrix of $A$ that lies in the rows and columns indexed by $\alpha$. A converse is presented that is the most general possible in terms of the data we use. Several corollaries are obtained by applying the main result to normal and Hermitian matrices. These corollaries lead to results concerning the case of equality in the interlacing inequalities for Hermitian matrices, and to the problem of the relationship among eigenvalue multiplicities in various principal submatrices.
\end{abstract}

Key words. interlacing inequalities, geometric multiplicity, principal submatrix, structured eigenvector

AMS subject classifications. $15 \mathrm{~A} 18,15 \mathrm{~A} 57$

For $\varnothing \neq \alpha \subseteq N \equiv\{1,2, \ldots, n\}$ and $A \in M_{n}(F)$, denote the principal submatrix of $A$ lying in the rows and columns indexed by $\alpha$ as $A[\alpha]$ and the complementary principal submatrix, resulting from the deletion of the rows and columns $\alpha$, as $A(\alpha)$. It is a straightforward partitioned matrix calculation that if $\lambda$ is an eigenvalue of $A, x$ an associated eigenvector, and $\alpha$ the set of positions in which $x$ has entries not equal to zero, then $\lambda$ is also an eigenvalue of $A[\alpha]$. Converses to this statement are known in certain special situations. For example, several people have recently noted that if $A \in M_{n}(C)$ is Hermitian, $|\alpha|=n-1$, and $\lambda \in R$ is an eigenvalue of both $A$ and $A[\alpha]$, i.e., a case of equality in the interlacing inequalities, then there is an eigenvector $x=\left(x_{1}, x_{2}, \ldots, x_{n}\right)^{T}$ of $A$ associated with $\lambda$ such that if $i \notin \alpha$ then $x_{i}=0$. For a general matrix $A \in M_{n}(F)$ and $\lambda$ an eigenvalue of $A$ with geometric multiplicity $k$, the rank of $A-\lambda I$ is $n-k$. Then for $|\alpha|>n-k$ the rank of $A[\alpha]-\lambda I$ is at most $n-k$ and $\lambda$ is also an eigenvalue of $A[\alpha]$. Moreover, it is implicit in the proof of Theorem 1.4.9 in [HJ] that there is an eigenvector of $A$ associated with $\lambda$ all of whose components indexed by $\alpha^{c}$ are zero. It is our purpose here to give a converse to the opening statement that is the most general possible in terms of the data we use. A variety of statements, including those just mentioned, may then be easily recognized as special cases.

The general converse, as well as some special cases, will be valid over a general field $F$. For $x \in F^{n}$ and $\alpha \subseteq N$, let $x[\alpha]$ be the subvector of $x$ containing the components of $x$ indexed by $\alpha$, and let $x(\alpha)$ be the complementary subvector. For $A \in M_{n}(F)$, let $\sigma(A)$ denote the set of all eigenvalues of $A$, some of which may lie only in an extension field of $F$, and for $\lambda \in \sigma(A)$, denote the geometric multiplicity of $\lambda$ in $A$ by $g_{\lambda}(A)$.

The most optimistic converse to the opening statement would be that if $\lambda$ is an eigenvalue of both $A$ and $A[\alpha]$, then there is an eigenvector $x$ (of $A$ associated with $\lambda)$ in which all components of $x(\alpha)$ are zero. However, this is not always the case.

* Received by the editors April 21, 1994; accepted for publication (in revised form) by T. Ando, July 22, 1994.

†Department of Mathematics, College of William and Mary, Williamsburg, Virginia 231878795. (kroschel@cs.wm.edu) The work of Dr. Johnson was supported, in part, by National Science Foundation grant DMS-92-00899 and Office of Naval Research contract N00014-90-J-1739. 
Consider

$$
A=\left[\begin{array}{cc:cc}
0 & 0 & 1 & 0 \\
0 & 1 & 0 & 0 \\
-1 & - & - & - \\
1 & 0 & 0 & 1 \\
0 & 0 & 1 & 0
\end{array}\right]
$$

and the set $\alpha=\{1,2\}$. This matrix has zero as an eigenvalue, as does $A[\alpha]$, but any eigenvector of $A$ associated with zero is of the form $\left[\begin{array}{llll}a & 0 & 0 & -a\end{array}\right]^{T}$. The converse cannot, therefore, be as general as one might hope.

Before stating a converse that is as general as it can be, several definitions are needed. The main result will be stated in terms of the dimensions of special subspaces, of the left and right eigenspaces of a general matrix $A$ associated with $\lambda$, in which the vectors have support among the components indexed by $\alpha$. These special subspaces (of the eigenspaces) are defined as follows:

$$
\begin{aligned}
& L E_{\alpha}^{\lambda}(A)=\left\{y \in F^{n} \mid y^{T} A=\lambda y^{T}, y(\alpha)=0\right\} \\
& R E_{\alpha}^{\lambda}(A)=\left\{x \in F^{n} \mid A x=\lambda x, x(\alpha)=0\right\}
\end{aligned}
$$

Similarly, let $L N(A)$ and $R N(A)$ denote the left and right nullspaces of $A$ and define the special subspaces (of the nullspaces) $L N_{\alpha}(A)=L E_{\alpha}^{0}(A)$ and $R N_{\alpha}(A)=R E_{\alpha}^{0}(A)$. It is clear that the dimensions of all these spaces are permutation similarity invariant, and this fact will be exploited repeatedly without further mention. If $x$ is an eigenvector of $A$ associated with $\lambda$, then $x$ is an eigenvector of $A-\lambda I$ associated with the eigenvalue zero. For this reason, results concerning the special nullspaces underlie observations concerning the special eigenspaces.

For contrast to the main result, we note some preliminary facts that indicate circumstances under which both the left and right special subspaces are nonempty. It is first observed that for general matrices, when the rank deficiency (the rank deficiency of a matrix $A$ is $\left.n-\operatorname{rank}(A)=g_{0}(A)\right)$ of a principal submatrix is sufficiently large, then the dimensions of the left and right nullspaces are positive. Suppose that the submatrix $A[\alpha]$ is such that its rank deficiency is greater than the number of rows or columns deleted from $A$ to obtain $A[\alpha]$. That is, for $|\alpha|=n-k, g_{0}(A[\alpha])>k$. In this case, the rank of $A[\alpha]$ is $n-k-g_{0}(A[\alpha])$ and the rank of $A$ can be at most $2 k$ more than the rank of $A[\alpha]$. But then the rank deficiency of $A$ is at least $g_{0}(A[\alpha])-k$. Since this number is positive, $A$ is rank deficient and the left and right nullspaces of $A$ are both nonempty. The lemma below states that, in fact, the left and right special nullspaces of $A$ are both nonempty.

Lemma 0. Let $A \in M_{n}(F)$ and let $\alpha \subseteq N$ be such that $|\alpha|=n-k$.

(i) If $g_{0}(A[\alpha])>k$, then $\operatorname{dim}\left(L N_{\alpha}(A)\right), \operatorname{dim}\left(R N_{\alpha}(A)\right) \geq g_{0}(A[\alpha])-k$.

(ii) Let $0 \leq g_{0} \leq \min \{k,|\alpha|\}$ be given. Then there is a matrix $B$ such that $g_{0}(B[\alpha])=g_{0}$ and $\operatorname{dim}\left(L N_{\alpha}(B)\right)=\operatorname{dim}\left(R N_{\alpha}(B)\right)=0$.

Proof. We assume, without loss of generality, that $\alpha=\{1,2, \ldots, n-k\}$. Then $A$ has the partitioned form

$$
A=\left[\begin{array}{ll}
A_{11} & A_{12} \\
A_{21} & A_{22}
\end{array}\right]
$$

in which $A_{11}=A[\alpha]$. In this case, if $x$ is in $R N_{\alpha}(A)$ it is of the form $x=\left[\begin{array}{l}\hat{x} \\ 0\end{array}\right]$ in which $\hat{x} \in F^{n-k}$. Similarly, any vector $y^{T} \in L N_{\alpha}(A)$ is of the form $y^{T}=\left[\begin{array}{ll}\hat{y}^{T} & 0\end{array}\right]$ in which $\hat{y} \in F^{n-k}$. 
Transformation of $A$ by an appropriate equivalence will not affect

$$
g_{0}(A), g_{0}(A[\alpha])=g_{0}\left(A_{11}\right)
$$

or the form of the nullvectors of $A$; so, choose $S, T \in M_{n-k}(F)$ nonsingular matrices such that

$$
\left[\begin{array}{ll}
S & 0 \\
0 & I
\end{array}\right]\left[\begin{array}{ll}
A_{11} & A_{12} \\
A_{21} & A_{22}
\end{array}\right]\left[\begin{array}{cc}
T & 0 \\
0 & I
\end{array}\right]=\left[\begin{array}{cc:c}
0 & 0 & Y_{1} \\
0 & I & Y_{2} \\
-\overline{X_{1}} & \overline{X_{2}} & \overline{A_{22}}
\end{array}\right]=\hat{A}
$$

in which the upper left zero block of $\hat{A}$ is $g_{0}\left(A_{11}\right)$-by- $g_{0}\left(A_{11}\right),\left[\begin{array}{c}Y_{1} \\ Y_{2}\end{array}\right]=S A_{12}$, and $\left[\begin{array}{ll}X_{1} & X_{2}\end{array}\right]=A_{21} T$. Because of the identity block in $\hat{A}$, a vector $x$ in $R N_{\alpha}(\hat{A})$ must be of the form

$$
x=\left[\begin{array}{c}
x_{1} \\
0 \\
0
\end{array}\right], x_{1} \in F^{g_{0}\left(A_{11}\right)} .
$$

In addition, $x_{1}$ must be in the right nullspace of the submatrix $X_{1}$. Conversely, for every vector in the right nullspace of $X_{1}$, there is a vector of the form indicated above in $R N_{\alpha}(\hat{A})$ and $\operatorname{dim}\left(R N_{\alpha}(\hat{A})\right)=\operatorname{dim}\left(R N\left(X_{1}\right)\right)$. Moreover, any vector in $R N_{\alpha}(\hat{A})$ corresponds to a vector in $R N_{\alpha}(A)$ of the form

$$
\left[\begin{array}{ll}
T & 0 \\
0 & I
\end{array}\right]\left[\begin{array}{c}
x_{1} \\
0 \\
0
\end{array}\right]=\left[\begin{array}{c}
T\left[\begin{array}{c}
x_{1} \\
0 \\
0
\end{array}\right]
\end{array}\right]=\left[\begin{array}{c}
\hat{x} \\
0
\end{array}\right]
$$

in which $\hat{x}=T\left[\begin{array}{l}x_{1} \\ 0\end{array}\right] \in F^{n-k}$. Therefore,

$$
\operatorname{dim}\left(R N_{\alpha}(A)\right)=\operatorname{dim}\left(R N_{\alpha}(\hat{A})\right)=\operatorname{dim}\left(R N\left(X_{1}\right)\right) .
$$

By similar arguments for the left nullspace

$$
\operatorname{dim}\left(L N_{\alpha}(A)\right)=\operatorname{dim}\left(L N_{\alpha}(\hat{A})\right)=\operatorname{dim}\left(L N\left(Y_{1}\right)\right) .
$$

A second equivalence will zero out $X_{2}$ and $Y_{2}$ :

$(2)$

$$
\left[\begin{array}{cc:c}
I & 0 & 0 \\
0 & I & 0 \\
-0 & -X_{2} & I
\end{array}\right]\left[\begin{array}{cc:c}
0 & 0 & Y_{1} \\
0 & I & Y_{2} \\
-X_{1} & -X_{2} & A_{22}
\end{array}\right]\left[\begin{array}{cc:c}
I & 0 & 0 \\
0 & I & -Y_{2} \\
-\overline{0} & - & - \\
0 & I
\end{array}\right]
$$

$$
=\left[\begin{array}{cc:c}
0 & 0 & Y_{1} \\
0 & I & 0 \\
-X_{1} & -0 & \frac{\tilde{A}_{22}}{2}
\end{array}\right]=\tilde{A}
$$

Note that this equivalence does not change the form of the nullvectors discussed above and the dimensional equalities still hold. 
Now, suppose that $g_{0}\left(A_{11}\right)>k$, as assumed in part (i) of the lemma. Since $Y_{1}$ and $X_{1}$ are $g_{0}\left(A_{11}\right)$-by- $k$ and $k$-by- $g_{0}\left(A_{11}\right)$, respectively,

$$
\operatorname{dim}\left(L N\left(Y_{1}\right)\right), \operatorname{dim}\left(R N\left(X_{1}\right)\right) \geq g_{0}\left(A_{11}\right)-k .
$$

But, $\operatorname{dim}\left(L N_{\alpha}(A)\right)=\operatorname{dim}\left(L N\left(Y_{1}\right)\right)$ and $\operatorname{dim}\left(R N_{\alpha}(A)\right)=\operatorname{dim}\left(R N\left(X_{1}\right)\right)$, so that part (i) of the lemma is verified.

For part (ii) consider the matrix

$$
B=\left[\begin{array}{ll}
B_{11} & B_{12} \\
B_{21} & B_{22}
\end{array}\right]=\left[\begin{array}{cc:cc}
0 & 0 & I_{g_{0}} & 0 \\
0 & I_{n-k-g_{0}} & 0 & 0 \\
- & - & - & - \\
I_{g_{0}} & 0 & * & 0 \\
0 & 0 & 0 & \hat{B}_{22}
\end{array}\right]
$$

in which $B_{11}$ is $(n-k)$-by- $(n-k)$ and $g_{0}\left(B_{11}\right)=g_{0}$. For this matrix, $0 \leq g_{0} \leq k$, but there are no nonzero vectors in either $L N_{\alpha}(B)$ or $R N_{\alpha}(B)$, and part (ii) of the lemma is also proved.

Replacement of $A$ with $A-\lambda I$ in Lemma 0 gives the following.

Theorem 0. Let $A \in M_{n}(F)$ and let $\alpha \subseteq N$ be such that $|\alpha|=n-k$.

(i) If $g_{\lambda}(A[\alpha])>k$, then $\operatorname{dim}\left(L E_{\alpha}^{\lambda}(A)\right), \operatorname{dim}\left(R E_{\alpha}^{\lambda}(A)\right) \geq g_{\lambda}(A[\alpha])-k$.

(ii) Let $0 \leq g_{\alpha} \leq \min \{k,|\alpha|\}$ be given. Then there is a matrix $B$ such that $g_{\lambda}(B[\alpha])=g_{\alpha}$ and $\operatorname{dim}\left(L E_{\alpha}^{\lambda}(B)\right)=\operatorname{dim}\left(R E_{\alpha}^{\lambda}(B)\right)=0$.

Statement (i) in Theorem 0 is best possible when left and right eigenspaces are considered separately. By considering the left and right eigenspaces simultaneously, one arrives at a general converse to the opening statement. This main result will first be stated in terms of the special nullspaces.

LEMmA 1. Let $A \in M_{n}(F)$; then for $\alpha \subseteq N$ with $|\alpha|=n-k$,

(i) $\operatorname{dim}\left(L N_{\alpha}(A)\right)+\operatorname{dim}\left(R N_{\alpha}(A)\right) \geq g_{0}(A)+g_{0}(A[\alpha])-k$.

(ii) Let $g$ and $g_{\alpha}$ such that $0 \leq g \leq n, 0 \leq g_{\alpha} \leq|\alpha|$, and $\left|g-g_{\alpha}\right| \leq k$ be given. Then, if $g+g_{\alpha}-k>0$ there is a matrix $B$ such that $g_{0}(B)=g, g_{0}(B[\alpha])=g_{\alpha}$ and

$$
\operatorname{dim}\left(L N_{\alpha}(B)\right)+\operatorname{dim}\left(R N_{\alpha}(B)\right)=g_{0}(B)+g_{0}(B[\alpha])-k .
$$

If $g+g_{\alpha}-k \leq 0$, then there is a matrix $B$, with the given parameters, such that

$$
\operatorname{dim}\left(L N_{\alpha}(B)\right)=\operatorname{dim}\left(R N_{\alpha}(B)\right)=0
$$

Proof. Begin the proof of Lemma 1 by performing the equivalences in (1) and (2) as in the proof of Lemma 0 . The matrices $Y_{1}$ and $X_{1}$ are of order $g_{0}\left(A_{11}\right)$-by- $k$ and $k$-by- $g_{0}\left(A_{11}\right)$, respectively. By basic linear algebra $\operatorname{dim}\left(L N\left(Y_{1}\right)\right)=g_{0}\left(A_{11}\right)-\operatorname{rank}\left(Y_{1}\right)$ and $\operatorname{dim}\left(R N\left(X_{1}\right)\right)=g_{0}\left(A_{11}\right)-\operatorname{rank}\left(X_{1}\right)$. Addition of these two equations results in

$$
\operatorname{dim}\left(L N\left(Y_{1}\right)\right)+\operatorname{dim}\left(R N\left(X_{1}\right)\right)=2 g_{0}\left(A_{11}\right)-\operatorname{rank}\left(Y_{1}\right)-\operatorname{rank}\left(X_{1}\right) .
$$

The equivalence transformations performed on $A$ in the proof of Lemma 0 do not change the rank of $A$ and, since

$$
\operatorname{rank}\left[\begin{array}{cc}
0 & Y_{1} \\
X_{1} & \tilde{A}_{22}
\end{array}\right] \geq \operatorname{rank}\left(Y_{1}\right)+\operatorname{rank}\left(X_{1}\right)
$$


we have

$$
\begin{aligned}
\operatorname{rank}(A)=\operatorname{rank}(\tilde{A}) & =\operatorname{rank}\left(A_{11}\right)+\operatorname{rank}\left[\begin{array}{cc}
0 & Y_{1} \\
X_{1} & \tilde{A}_{22}
\end{array}\right] \\
& \geq \operatorname{rank}\left(A_{11}\right)+\operatorname{rank}\left(Y_{1}\right)+\operatorname{rank}\left(X_{1}\right) .
\end{aligned}
$$

Combining (5) and (4) results in

$$
\begin{aligned}
\operatorname{dim}\left(R N\left(X_{1}\right)\right)+\operatorname{dim}\left(L N\left(Y_{1}\right)\right) & \geq 2 g_{0}\left(A_{11}\right)-\operatorname{rank}(A)+\operatorname{rank}\left(A_{11}\right) \\
& =g_{0}(A)+g_{0}\left(A_{11}\right)-k .
\end{aligned}
$$

From the discussion in the proof of Lemma $0 \operatorname{dim}\left(L N_{\alpha}(A)\right)=\operatorname{dim}\left(L N\left(Y_{1}\right)\right)$ and $\operatorname{dim}\left(R N_{\alpha}(A)\right)=\operatorname{dim}\left(R N\left(X_{1}\right)\right)$ so that

$$
\begin{aligned}
\operatorname{dim}\left(L N_{\alpha}(A)\right)+\operatorname{dim}\left(R N_{\alpha}(A)\right) & =\operatorname{dim}\left(L N\left(Y_{1}\right)\right)+\operatorname{dim}\left(R N\left(X_{1}\right)\right) \\
& \geq g_{0}(A)+g_{0}\left(A_{11}\right)-k,
\end{aligned}
$$

and part (i) of Lemma 1 is proved.

There are two cases to consider in proving part (ii) of Lemma 1. To begin, consider the case in which $g+g_{\alpha}-k \leq 0$. Note that for this to be the case, $g_{\alpha}$ must be less than or equal to $k$. For the matrix $B$ in (3), if $g_{\alpha}=g_{0}$, then $g_{0}\left(B_{11}\right)=g_{\alpha}$ and the submatrix $\hat{B}_{22}$ is $\left(k-g_{\alpha}\right)$-by- $\left(k-g_{\alpha}\right)$. This submatrix can be chosen so that $B$ has rank deficiency, $g$, from 0 to $k-g_{\alpha}$. Thus, $B$ has the appropriate parameters, and, as mentioned in the proof of Lemma $0, B$ has $\operatorname{dim}\left(L N_{\alpha}(B)\right)=\operatorname{dim}\left(R N_{\alpha}(B)\right)=0$.

For the case in which $g+g_{\alpha}-k>0$, consider

$$
B=\left[\begin{array}{cc|c}
0 & 0 & Y_{1} \\
0 & I_{n-k-g_{\alpha}} & 0 \\
\hline X_{1} & 0 & 0
\end{array}\right]
$$

The submatrices $Y_{1}$ and $X_{1}$ can independently be chosen to have rank from zero to $\min \left(g_{\alpha}, k\right)$, inclusive, which gives $B$ a rank deficiency, $g$, from $\left|g_{\alpha}-k\right|$ to $g_{\alpha}+k$, inclusive. Now, note that in (5) if $\tilde{A}_{22}=0$, then

$$
\operatorname{rank}(A)=\operatorname{rank}\left(A_{11}\right)+\operatorname{rank}\left(Y_{1}\right)+\operatorname{rank}\left(X_{1}\right)
$$

and there is equality in (6). Because $B$ is of this form, the equality holds and $\operatorname{dim}\left(L N_{\alpha}(B)\right)+\operatorname{dim}\left(R N_{\alpha}(B)\right)=g_{0}(B)+g_{0}\left(B_{11}\right)-k$, which proves the lemma.

Our main result, the proof of which follows from Lemma 1 by translation, is then:

TheOREM 1. Let $A \in M_{n}(F)$; then for $a \subseteq N$ with $|\alpha|=n-k$

(i) $\operatorname{dim}\left(L E_{\alpha}^{\lambda}(A)\right)+\operatorname{dim}\left(R E_{\alpha}^{\lambda}(A)\right) \geq g_{\lambda}(A)+g_{\lambda}(A[\alpha])-k$.

(ii) Let $g$ and $g_{\alpha}$ such that $0 \leq g \leq n, 0 \leq g_{\alpha} \leq|\alpha|$, and $\left|g-g_{\alpha}\right| \leq k$ be given. Then, if $g+g_{\alpha}-k>0$ there is a matrix $B$ such that $g_{\lambda}(B)=g, g_{\lambda}(B[\alpha])=g_{\alpha}$ and

$$
\operatorname{dim}\left(L E_{\alpha}^{\lambda}(B)\right)+\operatorname{dim}\left(R E_{\alpha}^{\lambda}(B)\right)=g_{\lambda}(B)+g_{\lambda}(B[\alpha])-k .
$$

If $g+g_{\alpha}-k \leq 0$, then there exists a matrix $B$, with the given parameters, such that

$$
\operatorname{dim}\left(L E_{\alpha}^{\lambda}(B)\right)=\operatorname{dim}\left(R E_{\alpha}^{\lambda}(B)\right)=0 .
$$


In each of Lemmas 0 and 1 and Theorems 0 and 1, statement (ii) indicates that statement (i) is best possible. The restrictions regarding $\alpha$ only avoid logical impossibilities and, otherwise, all situations not covered by statement (i) are covered in statement (ii).

At this point we make two general observations that are direct consequences of Theorem 1.

(i) If $A \in M_{n}(F)$ and $|\alpha|=n-1$, then $\lambda \in \sigma(A) \cap \sigma(A[\alpha])$ if and only if there is either a left or a right eigenvector of $A$ (associated with $\lambda$ ) whose $\alpha^{c}$ component is zero.

(ii) If $A \in M_{n}(F), \lambda \in \sigma(A)$ and $\alpha \subseteq N$ with $|\alpha|=n-k$ are such that $\operatorname{dim}\left(L E_{\alpha}^{\lambda}(A)\right)=\operatorname{dim}\left(R E_{\alpha}^{\lambda}(A)\right)$, then each of

$$
\operatorname{dim}\left(L E_{\alpha}^{\lambda}(A)\right), \operatorname{dim}\left(R E_{\alpha}^{\lambda}(A)\right) \geq \frac{g_{\lambda}(A)+g_{\lambda}(A[\alpha])-k}{2} .
$$

In this event, if $g_{\lambda}(A)+g_{\lambda}(A[\alpha])>k$, then both $\operatorname{dim}\left(L E_{\alpha}^{\lambda}(A)\right)$ and $\operatorname{dim}\left(R E_{\alpha}^{\lambda}(A)\right)$ are positive.

Note that statement (i) does not follow from Theorem 0 and that statement (i) cannot be improved, as it may be that there is not both a left special eigenvector and a right special eigenvector. For example,

$$
A=\left[\begin{array}{ccc}
1 & -1 & 0 \\
-1 & 1 & 0 \\
0 & -1 & 1
\end{array}\right]
$$

does not have the property assumed in (ii) for $0 \in \sigma(A)$, and $g_{0}(A)=1=g_{0}(A[\{1,2\}])$. Thus, as every right null vector of $A$ is a multiple of $(1,1,1)^{T}, A$ has no special right eigenvector associated with 0 , while it, of course, has a left such eigenvector, e.g., $(1,1,0)$, because of statement (i). Similarly, for many values of $g_{\lambda}(A)$ and $g_{\lambda}(A[\alpha])$, the conclusion of (ii) does not follow from Theorem 0 , and, for further values, the estimates that follow from Theorem 0 are weaker. For example, the statement about Hermitian matrices in the opening paragraph does not follow from Theorem 0.

We may now give several specific corollaries to Theorem 1. First, note that if $A \in M_{n}(C)$ is normal, then, as $U A U^{*}=D$, with $U$ unitary and $D$ diagonal, any left eigenspace of $A$ is the conjugate transpose of a right eigenspace. Thus, the hypothesis of (ii) above is satisfied for each $\lambda$ and $\alpha$. From this observation we can conclude the following.

CoRollary 1. Let $A \in M_{n}(C)$ be a normal matrix. For $\alpha \subseteq N$ with $|\alpha|=n-k$

$$
\operatorname{dim}\left(L E_{\alpha}^{\lambda}(A)\right), \operatorname{dim}\left(R E_{\alpha}^{\lambda}(A)\right) \geq \frac{g_{\lambda}(A)+g_{\lambda}(A[\alpha])-k}{2} .
$$

Of course Hermitian matrices are normal so the following is a special case of Corollary 1.

Corollary 2. Let $A \in M_{n}(C)$ be Hermitian. For $\alpha \subseteq N$ with $|\alpha|=n-k$

$$
\operatorname{dim}\left(L E_{\alpha}^{\lambda}(A)\right), \operatorname{dim}\left(R E_{\alpha}^{\lambda}(A)\right) \geq \frac{g_{\lambda}(A)+g_{\lambda}(A[\alpha])-k}{2} .
$$

In the opening paragraph we mentioned that if $A$ is Hermitian, $\lambda \in \sigma(A) \cap \sigma(A[\alpha])$, and $|\alpha|=n-1$, then there is an eigenvector $x$ (of $A$ associated with $\lambda$ ) in which 
$x(\alpha)=0$. But then $g_{\lambda}(A), g_{\lambda}(A[\alpha]) \geq 1$ which results in a positive right-hand side in Corollary 2. In this case, both the left and the right special eigenspaces are nonempty, which proves the following corollary.

Corollary 3. Let $A \in M_{n}(C)$ be Hermitian, let $\alpha \subseteq N$ be such that $|\alpha|=n-1$, and let $\lambda \in R$ be an eigenvalue of $A$. Then, there is an eigenvector $x$ of $A$ associated with $\lambda$ such that $x(\alpha)=0$ if and only if $\lambda \in \sigma(A[\alpha])$.

Thus, the general scheme adopted here provides an algebraic proof to the statement in the opening paragraph.

In the case that $A$ is Hermitian, the interlacing inequalities [HJ, Thm. 4.3.8] hold and, since any principal submatrix of an Hermitian matrix is Hermitian, Corollary 3 may be applied at each "level" of interlacing. Sequential application of Corollary 3 will lead to the corollaries below, but first several definitions are needed. For the following discussion, let $A \in M_{n}(C)$ be Hermitian. Suppose $\lambda$ is an eigenvalue of $A$, then $A$ is said to have interlacing equality at $\lambda$ of breadth $k$ if there are exactly $k$ distinct index sets $\alpha_{1}, \alpha_{2}, \ldots, \alpha_{k} \subseteq N$ in which $\left|\alpha_{i}\right|=n-1$ and $\lambda \in \sigma\left(A\left[\alpha_{i}\right]\right), i=1,2, \ldots, k$. If $A$ is such that $g_{\lambda}(A)=1$, then the breadth of interlacing equality at $\lambda$ is just the number of zero components in an eigenvector (because of Corollary 3 ). The matrix $A$ is said to have interlacing equality at $\lambda$ of depth $k$ if $\lambda \in \sigma\left(A\left[\beta_{j}\right]\right)$ for some index sets $\beta_{0}, B_{1}, \ldots, B_{k} \subseteq N$ such that $\beta_{j+1} \subset \beta_{j}, j=0,1, \ldots, k-1,\left|\beta_{j}\right|=n-j, j=0,1, \ldots, k$ and $k$ is a maximum. If, in addition, $g_{\lambda}\left(A\left[\beta_{j+1}\right]\right) \geq g_{\lambda}\left(A\left[\beta_{j}\right]\right), j=0,1, \ldots, k-1$, then $A$ is said to have interlacing equality at $\lambda$ of restricted depth $k$. Here, $k$ is the number of principal submatrices in the nested sequence for which the geometric multiplicity of $\lambda$ is nondecreasing, so that the depth of interlacing equality may be greater than the restricted depth. The following corollaries relate these concepts.

Corollary 4. Let $A \in M_{n}(C)$ be Hermitian and be such that $g_{\lambda}(A)=1$. If $A$ has interlacing equality at $\lambda$ of breadth $k$, then $A$ has interlacing equality at $\lambda$ of depth at least $k$.

Proof. If $A$ has interlacing equality at $\lambda$ of breadth $k$, then there are $k$ distinct principal submatrices $A\left[\alpha_{i}\right]$ such that $\lambda \in \sigma\left(A\left[\alpha_{i}\right]\right)$ and $\left|\alpha_{i}\right|=n-1$. In this case, $g_{\lambda}\left(A\left[\alpha_{i}\right]\right) \geq 1$ and, by assumption, $g_{\lambda}(A)=1$. Thus, by Corollary 3 , for each $\alpha_{i}$ there is an eigenvector $y_{i}$ of $A$ associated with $\lambda$, such that $y_{i}\left(\alpha_{i}\right)=0$. However, since $g_{\lambda}(A)=1$, the (right) eigenspace of $A$ associated with $\lambda$ is one dimensional, so that each of the $y_{i}$ 's may be taken to be the same, $x$. It follows that $x\left(\alpha_{1} \cap \cdots \cap \alpha_{k}\right)=0$. By the partitioned calculation mentioned in the opening paragraph $\beta_{0}=N$, and $\beta_{i}=\alpha_{1} \cap \cdots \cap \alpha_{i}, i=1, \ldots, k$, exhibit that $A$ has interlacing equality at $\lambda$ of depth at least $k$.

Corollary 4 is stated in the Hermitian case for parallelism to the corollaries that follow. However, it should be noted that the argument is equally valid in the normal case (using Corollary 1 in place of Corollary 3 with an obvious generalization of the definitions), so that Corollary 4 may be generalized by replacing "Hermitian" in the hypothesis with "normal." On the other hand, Corollary 4 is not valid for general matrices, as exhibited by the example

$$
\left[\begin{array}{lll}
1 & 1 & 1 \\
1 & 1 & 1 \\
0 & 1 & 1
\end{array}\right]
$$

in which 0 is an eigenvalue of breadth 2 , while its depth is only 1 .

The converse to Corollary 4 does not hold. A counterexample is given by the 
matrix

$$
A=\left[\begin{array}{llll}
0 & 0 & 1 & 1 \\
0 & 0 & 1 & 1 \\
1 & 1 & 1 & 1 \\
1 & 1 & 1 & 0
\end{array}\right]
$$

which has interlacing equality at 0 of depth $3(A(\{4\}), A(\{3,4\}), A(\{2,3,4\}))$, but interlacing equality at 0 of breadth only $2(A(\{3\}), A(\{4\}))$. However, the geometric multiplicities of the principal submatrices that yield interlacing equality at 0 of depth 3 are

$$
\begin{aligned}
& g_{0}(A(\{4\}))=1, \\
& g_{0}(A(\{3,4\}))=2, \\
& g_{0}(A(\{2,3,4\}))=1 .
\end{aligned}
$$

In fact, the restricted depth of interlacing equality at 0 is only 2 and this is exactly the breadth of interlacing equality at 0 . As indicated in the following corollary, the breadth of interlacing equality at $\lambda$ must be at least that of the restricted depth.

Corollary 5. Let $A \in M_{n}(C)$ be Hermitian and suppose $\lambda \in \sigma(A)$. If $A$ has interlacing equality at $\lambda$ of restricted depth $k$, then $A$ has interlacing equality at $\lambda$ of breadth at least $k$.

Proof. If $g_{\lambda}(A)>1$, the breadth at $\lambda$ is $n$ (see discussion later, if necessary) and the conclusion is automatically valid. Thus, we suppose $g_{\lambda}(A)=1$. If $A$ has interlacing equality at $\lambda$ of restricted depth $k$, then there is some nested sequence of $k+1$ principal submatrices $A\left[\beta_{i}\right]$, such that $\left|\beta_{i}\right|=n-i, \lambda \in \sigma\left(A\left[\beta_{i}\right]\right), i=0,1, \ldots, k$, and $g_{\lambda}\left(A\left[\beta_{i+1}\right]\right) \geq g_{\lambda}\left(A\left[\beta_{i}\right]\right), i=0,1, \ldots, k-1$. Assume, without loss of generality, that the rows and columns of $A\left[\beta_{i}\right]$ are numbered 1 to $n-i$. Note that $n-i$ is the index of the row and column deleted from $A\left[\beta_{i}\right]$ to obtain $A\left[\beta_{i+1}\right]$. By Corollary 2

$$
\begin{aligned}
\operatorname{dim}\left(L E_{\beta_{i+1}}^{\lambda}\left(A\left[\beta_{i}\right]\right)\right), \operatorname{dim}\left(R E_{\beta_{i+1}}^{\lambda}\left(A\left[\beta_{i}\right]\right)\right) & \geq \frac{g_{\lambda}\left(A\left[\beta_{i}\right]\right)+g_{\lambda}\left(A\left[\beta_{i+1}\right]\right)-1}{2} \\
& \geq g_{\lambda}\left(A\left[\beta_{i}\right]\right)-\frac{1}{2}
\end{aligned}
$$

since $g_{\lambda}\left(A\left[\beta_{i}\right]\right) \leq g_{\lambda}\left(A\left[\beta_{i+1}\right]\right)$. Both dimensions must be integral; so, the dimensions of the special eigenspaces must both be at least $g_{\lambda}\left(A\left[\beta_{i}\right]\right)$. Then, every (left and right) eigenvector of $A\left[\beta_{i}\right]$ associated with $\lambda$ is in the special (left and right) eigenspace and, thus, component $n-i$ of each of these vectors is 0 .

Let $x$ be an eigenvector (essentially unique) of $A$ associated with $\lambda$. Since

$$
g_{\lambda}(A)=g_{\lambda}\left(A\left[\beta_{0}\right]\right)=1 \quad \text { and } \quad g_{\lambda}\left(A\left[\beta_{1}\right]\right) \geq 1,
$$

by Corollary $3, x\left(\beta_{1}\right)=0$. By the preceding paragraph, if $i=1$, then every eigenvector of $A\left[\beta_{1}\right]$ associated with $\lambda$, including $x\left[\beta_{1}\right]$, has a zero in the $n-1$ component. Thus, $x\left(\beta_{1} \cap \beta_{2}\right)=x\left(\beta_{2}\right)=0$.

Continuing in this manner, for each $i=0,1, \ldots, k-1, x\left[\beta_{i}\right]$ is an eigenvector of $A\left[\beta_{i}\right]$ associated with $\lambda$ with a zero in the $n-i$ component so that

$$
x\left(\beta_{1} \cap \beta_{2} \cap \cdots \cap \beta_{i+1}\right)=x\left(\beta_{i+1}\right)=0 .
$$

Then, $x\left(\beta_{k}\right)=0$ and for each $j \notin \beta_{k}, x(\{j\})$ is an eigenvector of $A(\{j\})$ associated with $\lambda$. Thus, $\alpha_{j}=N-\{n+1-j\}, j=1, \ldots, k$, exhibits that $A$ has interlacing equality at $\lambda$ of breadth at least $k$. 
Note that the breadth of interlacing equality can be strictly greater than the restricted depth of interlacing equality. For example, the matrix

$$
\left[\begin{array}{lll}
0 & 0 & 1 \\
0 & 0 & 0 \\
1 & 0 & 0
\end{array}\right]
$$

has interlacing equality at 0 of restricted depth 1 , but the breadth of interlacing equality at 0 is 2 .

If the matrix $A$ is such that $g_{\lambda}(A[\alpha]) \leq 1$ for every index set $\alpha \subseteq N$, and $A$ has interlacing equality at $\lambda$ of $\operatorname{depth} k$, then $A$ also has interlacing equality at $\lambda$ of restricted depth $k$. In this case, by Corollary $5, A$ has interlacing equality at $\lambda$ of breadth at least $k$. Combining Corollaries 4 and 5 then yields the following.

COROLlaRY 6. Let $A \in M_{n}(C)$ be Hermitian and suppose for every index set $\alpha \subseteq N$ that $g_{\lambda}(A[\alpha]) \leq 1$ with $g_{\lambda}(A)=1$. Then, $A$ has interlacing equality at $\lambda$ of breadth $k$ if and only if $A$ has interlacing equality at $\lambda$ of depth $k$.

Let $A \in M_{n}(C)$ be Hermitian. Due to classical interlacing, when $g_{\lambda}(A)>1, \lambda \in$ $\sigma(A[\alpha])$ for any $\alpha \subseteq N$ such that $|\alpha|=n-1$. In addition, when $g_{\lambda}(A)>1$ there is for each such $\alpha$ an eigenvector, $z$, of $A$ associated with $\lambda$ such that $z(\alpha)=0$. This may be seen in an elementary way by noting that, given any two linearly independent eigenvectors $x, y$ in the eigenspace, there is a linear combination with a zero in any specified position. Such an $A$ has interlacing equality at $\lambda$ of breadth $n$, but may have depth at $\lambda$ as little as 1 . For example, the matrix

$$
\left[\begin{array}{lll}
1 & 1 & 1 \\
1 & 1 & 1 \\
1 & 1 & 1
\end{array}\right]
$$

has interlacing equality at 0 of breadth 3 , while the depth at 0 is only 1 . Thus, the assumption in Corollaries 4 and 6 that $g_{\lambda}(A)=1$ is necessary.

\section{REFERENCES}

[HJ] R. Horn And C. R. Johnson Matrix Analysis, Cambridge University Press, Cambridge, 1985. 
Reproduced with permission of the copyright owner. Further reproduction prohibited without permission. 